
gas natural.

\title{
CAPITAL TRANSNACIONAL Y GOBERNANZA ENERGÉTICA EN LA UNIÓN EUROPEA. EL CASO DEL GAS NATURAL
}

\section{TRANSNATIONAL CAPITAL AND ENERGY GOVERNANCE IN THE EUROPEAN UNION. THE CASE OF NATURAL GAS}

\author{
ANA MORENO \\ Universidad del País Vasco \\ ana.morenor@ehu.eus
}

Fecha de recepción: mayo 2016 Fecha de aceptación: noviembre 2016

\begin{abstract}
RESUMEN
El principal objetivo de este artículo es analizar la liberalización del sector del gas natural en Europa. En cuanto a la política energética, no podemos considerar a la Unión Europea o sus Estados Miembro como simples agentes del capital. Sin embargo, otorgarles una autonomía según la cual éstos desarrollan sus propios intereses nos situaría en un escenario en el que se minimiza el papel del capital. Así pues, esta investigación sigue el trabajo de la Escuela de Ámsterdam sobre la formación transnacional de clase. Esta aproximación subraya la necesidad de la clase dominante de ejercer una agencia colectiva para reproducir su dominio y asegurar que éste se articula en políticas concretas
\end{abstract}

PALABRAS CLAVE: Capital transnacional, gobernanza energética, Unión Europea, gas natural, mercado interior.

\begin{abstract}
The main objective of this paper is to analyze the liberalization of natural gas sector in Europe. Regarding the energy policy, we cannot consider the EU or its Member States just as agents of capital. However, granting them an autonomy according to which they develop their own interests, would lead us into a scenario that minimizes the role of capital. This research, then, follows the work of the Amsterdam School about transnational class formation. This approach underlines the need of the dominant class of exercising a collective agency to reproduce their dominance and to ensure that it articulates in concrete politics.
\end{abstract}

KEY WORDS: Transnational capital, energy governance, European Union, natural gas, internal market.

JEL: F59, P16, P18, Q48

\section{INTRODUCCIÓN}

El principal objetivo de este trabajo es analizar la liberalización del sector del gas natural en Europa. La estrategia política europea que, en términos ideales, estaría 
Moreno, Ana. Capital transnacional y gobernanza energética en la Unión Europea. El caso del gas natural.

dirigida a paliar la situación de dependencia exterior de la UE en cuanto al abastecimiento de hidrocarburos, recoge resultados contradictorios. Entonces, lo que propongo es un recorrido a través del proceso de construcción de esta estrategia que nos permita descubrir su origen social, su objetivo último, así como el papel que juegan los distintos grupos sociales y en qué lugar quedan estos como consecuencia de su implementación.

Respecto a la política energética, no podemos considerar a la Unión Europea (UE) o a sus estados miembro (EEMM) como simples agentes del capital. Sin embargo, otorgarles una autonomía según la cual éstos desarrollan sus propios intereses vinculados a la supervivencia en un sistema internacional anárquico, minimiza el papel del capital relegándolo a un plano secundario. Por el contrario, en este estudio las relaciones sociales se presentan como elemento clave para el análisis. La actividad de los estados se examina reconociéndolos dentro del marco de lo que Cox denomina "States-society complexes" (Cox, 1981), y teniendo en cuenta que con el despliegue del capitalismo global, las relaciones sociales basadas en una distribución desigual del poder económico, político y social, que dan sentido al concepto, transcienden el ámbito estatal, aplicamos el mismo criterio para la UE.

Así, esta investigación, siguiendo el trabajo en EPI de la Escuela de Ámsterdam, subraya la naturaleza de clase de la sociedad capitalista vinculándola al análisis de la clase capitalista como clase dominante. La transnacionalización del capitalismo global ha supuesto una progresiva transnacionalización de las fuerzas sociales y más concretamente, de aquellas vinculadas con el capital transnacional. Los principales intereses de la clase capitalista transnacional, surgida de este proceso, están vinculados a la transnacionalización y liberalización de la economía política global (Gill, 2008:93). Si bien resulta evidente el poder estructural del capital transnacional, basado principalmente en su movilidad, también hay que tener en cuenta que la clase dominante debe actuar de forma colectiva a fin de reproducir su dominio y asegurarse de que éste se articula en políticas concretas (Van Apeldoorn, 2002: 26). Su acción colectiva es posible gracias a un proceso de organización que ha permitido su establecimiento como actor político de pleno derecho. En el contexto europeo se ha identificado un bloque de poder transnacional dirigido por una élite capitalista (no necesariamente interna a la UE) aliada con ciertos elementos de la Comisión Europea (Van Apeldoorn, 2000: 158-9).

Desde los primeros años de la década de 1990, la emergencia de de una ideología neoliberal, de inspiración anglosajona, en la economía política europea daba paso a las políticas de liberalización económica y privatización de las empresas estatales que facilitarían la reforma del sector gasístico. El nuevo modelo, basado en el corto plazo, los mercados de spot y los diferentes instrumentos financieros, incorporaba nuevos y poderosos actores con sus propios intereses, concretamente, aquellos relacionados con la desregulación y liberalización de los mercados del gas europeos (Fernández y Palazuelos, 2014: 6).

En este sentido, el discurso político neoliberal viene marcado por los conceptos de eficiencia y competitividad como las mayores ventajas de la liberalización. 
Moreno, Ana. Capital transnacional y gobernanza energética en la Unión Europea. El caso del gas natural.

Pero en contra de la propuesta académica liberal sobre una sociedad transnacional basada en los efectos (el spill over) que pudieran traspasarse de las instituciones internacionales o supranacionales hacia la sociedad, en la práctica, las reformas en el mercado del gas han llevado a una profundización en el modelo oligopólico de etapas anteriores (Fernández y Palazuelos, 2014: 14). Ésta es una cuestión que, desde una postura crítica, refleja la importancia de las relaciones de poder en la estructura de relaciones sociales de la UE y el hecho de que las reformas se construyen en línea con el capital transnacional implicado en el sector. Los acontecimientos que se están desarrollando alrededor del modelo de suministro de gas en Europa, sólo se pueden explicar si tenemos en cuenta estas dinámicas.

El artículo, basado principalmente en el análisis de fuentes secundarias, se estructura de la siguiente manera. Después de esta introducción, en la que he contextualizado el problema principal de la investigación mediante una breve revisión bibliográfica y descrito someramente el enfoque teórico del estudio, pasaré a detallar esto último con mayor profundidad. A continuación, analizaré la construcción y el desarrollo del modelo europeo del gas en las últimas décadas del siglo XX. Después examinaré las condiciones que permitieron su cambio hacia la liberalización para finalizar realizando un breve recorrido por sus primeros resultados. Para terminar, expondré unas breves conclusiones.

\section{MARCO TEÓRICO}

El debate académico sobre las políticas de energía, en gran parte gira alrededor del debate más general entre el neorrealismo y el institucionalismo neoliberal (Correljé, y Van Der Linde, 2006; Youngs, 2007). Como ya se ha sugerido, la centralidad del estado en el análisis neorrealista soslaya la actuación de las fuerzas sociales en el plano transnacional. La aproximación neoliberal, por su parte, destaca el papel que juegan las instituciones internacionales y los mercados de la energía como actores importantes. Sin embargo, subordina la actuación de otros actores transnacionales a la iniciativa de estas instituciones. Así, en el contexto europeo los grupos empresariales transnacionales actuarían en respuesta a los cambios promovidos desde la Comisión Europea (Van Apeldoorn, 2002: 37-8). Desde el primer enfoque, la energía se sitúa en el ámbito de la lucha por el poder (Ciuta, 2010: 130) y su importancia reside en lo mucho que afecta a la distribución de recursos en el sistema internacional (Ciuta, 2010: 130). A este respecto, Stoddar (2013: 444) refiere el argumento de Waltz (particularmente adecuado en nuestro caso) señalando que, en aras de garantizar la seguridad, los estados deben "to control what they depend on or to lessen the extent of their dependency". La aproximación neoliberal, por otra parte, se basa en la existencia de mercados e instituciones efectivos (Correljé, y Van Der Linde, 2006: 1). Desde este punto de vista, la politización de la energía (es decir, la intervención del estado) es lo que conduce a una escasez que de otro modo podría solucionarse por medio de la innovación tecnológica, el descubrimiento de nuevos depósitos y el desarrollo de fuentes alternativas (Ciuta, 2010: 134).

Desarrollando el razonamiento neorrealista, la energía, y en concreto los hidrocarburos, tienen la potencialidad de convertirse tanto en la causa como en el instrumento en una guerra o conflicto (ver por ejemplo Ciuta, 2010; Mansson, 2014): 
Moreno, Ana. Capital transnacional y gobernanza energética en la Unión Europea. El caso del gas natural.

en el primer caso, el control de los hidrocarburos y el acceso a ellos se considera una causa probable de conflicto entre las grandes potencias, y en el segundo, se plantea lo que se conoce como energy weapon, es decir, la capacidad que tendrían algunos estados de utilizar la energía como un medio para conseguir sus objetivos políticos en otros ámbitos. Bajo el criterio institucionalista, sin embargo, la interdependencia existente en los mercados de la energía reduce las posibilidades de desarrollo de conflictos (por ejemplo, Truscott, 2009: 31). Se excluye la dimensión del poder en el análisis puesto que el mercado, desde esta óptica, se entiende como un conjunto de intercambios mutuamente beneficiosos, que no necesitan del ejercicio del poder (Stoddard, 2013; 446). La gestión internacional cooperativa de la energía a través de regímenes posibilitaría el establecimiento de mejores precios gracias a la optimización de los costes de transacción y la mejora de la información (Hancock y Vivoda, 2014: 208).

El problema que nos plantean estas propuestas se refiere a la división estadomercado que se extrae de ambos planteamientos. Así, mientras el neorrealismo y el neoliberalismo soslayan el papel de las fuerzas sociales y las relaciones sociales que impulsan, sostienen y transforman los estados y los mercados (De Graaf, 2013: 45), si lo que buscamos es entender la definición de las políticas de la energía en la Unión Europea (como forma sui generis de estado ${ }^{1}$ ), conviene realizar un recorrido a través de su proceso de construcción, visto como resultado de la interacción entre esas fuerzas sociales en la arena política que representa la UE ${ }^{2}$ (Van Apeldoorn, 2002). Para ello, aquí partimos de un reconocimiento de la estructura de gobernanza y autoridad política que posee la UE. Pese a que no pueda ser considerada como una réplica del estado nacional a un nivel supranacional, esta estructura debería estar sostenida por una cierta configuración de fuerzas sociales. En este punto, siguiendo el trabajo de la Escuela de Ámsterdam en EPI sobre la formación transnacional de clase (Van der Pijl, 1998; Van Apeldoorn, 2004), se plantea un reconocimiento de la naturaleza de clase de la sociedad capitalista vinculado al dominio de la clase capitalista como clase dirigente, y es la clase lo que aquí se considera como la conexión entre la formación de las estrategias políticas de la UE y el capital.

Un segundo problema tiene que ver con el planteamiento que se realiza de la gobernanza de la energía. Las definiciones de gobernanza a menudo se basan en premisas liberales sobre el papel que juega la cooperación en el logro de objetivos comunes en un mundo interdependiente. Sin embargo, una perspectiva crítica no puede más que subrayar la desigual distribución de poder y oportunidades entre los diferentes actores participantes. Desde este ángulo, las prácticas dominantes de la gobernanza actual son principalmente un medio para que ciertos actores 0 colectivos impongan un conjunto de reglas de las cuales ellos mismos se benefician o que expresan sus ideas e intereses a expensas de los grupos menos poderosos de la sociedad global (Overbeek, 2010). Así, siguiendo a Van Apeldoorn y De Graaff (2012), planteo que tanto la UE como sus EEMM son estructuras que forman una

1 Van Apeldoorn sugiere que en realidad representa una nueva forma de dominación política debido a la ausencia del monopolio de la violencia que tradicionalmente define al estado.

2 En su texto, el autor se refiere al contenido socioeconómico global de la integración europea. Sin embargo, parece conveniente aplicar el mismo criterio a nuestro campo concreto. 
Moreno, Ana. Capital transnacional y gobernanza energética en la Unión Europea. El caso del gas natural.

arena política donde las fuerzas sociales promueven sus propias estrategias y proyectos políticos. A su vez, esta arena está estructuralmente predispuesta a seleccionar las estrategias de unas clases (o fracciones de clase) sobre las de otras (Van Apeldoorn y De Graaff, 2012: 5). Por tanto, el problema que aquí se plantea es que los hidrocarburos están rodeados de un conglomerado de intereses privados que se pueden convertir en obstáculos para el desarrollo de una gobernanza adecuada de la energía. Entre estos intereses, los más importantes son los de las élites relacionadas con las grandes compañías del sector.

\subsection{Estados, UE y empresas transnacionales en la política de los hidrocarburos}

Tanto los estados como la UE pueden jugar diferentes papeles en relación con el proceso de acumulación capitalista: pueden dirigir los mercados, crearlos, corregirlos y representar los intereses del capital "interno" en el exterior (Van Apeldoorn, De Graaff y Overbeek 2012: 473-6). Pero en el sector de los hidrocarburos, estas dinámicas de interacción entre el capital y el poder territorial se multiplican: por un lado, los recursos están territorialmente fijados, los hidrocarburos son fundamentales para la producción, tienen importantes efectos macroeconómicos y están en la base de cualquier aparato militar. Además, hay que tener en cuenta que muchas de las importaciones europeas provienen de territorios donde el estado, además de la propiedad del producto en sí, tiene la propiedad total o parcial de las empresas que se encargan de su producción (De Graaff, 2013: 40). Sin embargo, el capital que debe aplicarse a los recursos para que estos obtengan valor no se encuentra necesariamente vinculado a un territorio y los mercados transnacionales introducen dinámicas y actores con sus propios intereses (como la financiarización y la especulación). Finalmente, hay que señalar el entramado de tratados en los que se apoyan los mercados, así como el respaldo militar que se les aplica (De Graaff, 2013: 54).

En este sentido, para comprender los movimientos de interacción que tienen lugar entre el capital y el territorio en el sector de la energía es necesario superar la dicotomía que se ha planteado entre las lógicas que dirigen cada uno de estos ámbitos $^{3}$. Así, lo que aquí se plantea es que las estrategias políticas de los estados y la UE están siempre mediadas por unas fuerzas sociales, que además no son necesariamente internas al territorio (Van Apeldoorn, 2000: 158-9). Van Apeldoorn, por ejemplo, propone sustituir el examen de la relación dialéctica entre el capital y el territorio por el análisis de la agencia de clase capitalista. Partiendo del reconocimiento del poder estructural del capital y en concreto la dependencia del estado del éxito de la acumulación capitalista, el autor recuerda que el dominio de la clase capitalista no está determinado por la estructura (aunque, le sea favorable) sino que debe realizar ciertos esfuerzos para asegurar su reproducción (Van Apeldoorn, 2000: 159).

Entonces, para entender la formación de la política transnacional de la UE tanto dentro como fuera de su territorio, tenemos que integrar estructura y agencia en la

3 Harvey plantea que la lógica del capital y la lógica territorial del poder deben verse como distintas la una de la otra, aunque entrelazadas de manera dialéctica (Harvey, 2003: 29-30). 
Moreno, Ana. Capital transnacional y gobernanza energética en la Unión Europea. El caso del gas natural.

explicación del dominio de la clase capitalista. El concepto neo-gramsciano de proyecto hegemónico, como veremos, resulta útil para el análisis de la instauración del orden energético europeo después de la II Guerra Mundial. Un proyecto hegemónico se puede entender como una orientación estratégica por parte de una clase o fracción de clase aspirante a la hegemonía ${ }^{4}$. Para que el proyecto hegemónico consiga traducirse en políticas y códigos institucionalizados en los estados debe conseguir articularse primero, con los intereses potencialmente divergentes que puedan existir dentro de la misma clase capitalista y segundo, integrar las reivindicaciones de las clases subordinadas que le sean asumibles de manera que pueda llegar a presentarse, en última instancia, como reflejo del bien común. La estructura de la UE ha facilitado a la clase capitalista transnacional la constitución de un proyecto en un espacio social transnacional. Sin embargo, el déficit democrático que sufre la UE (Van Apeldoorn, 2000: 160) que se refleja en unas clases subordinadas alejadas de los espacios de decisión y que encuentran serias dificultades para exigir responsabilidad democrática, mientras se incrementa el poder de las fuerzas vinculadas al capital transnacional, ha ido convirtiendo en innecesaria o al menos, relegando a un plano muy secundario esta articulación transversal de intereses dirigida a alcanzar la hegemonía.

Así pues, en lo que se refiere a la situación actual, parece más adecuado trabajar desde el concepto de nuevo constitucionalismo acuñado por Gill. Éste se refiere a la construcción de mecanismos legales o constitucionales dirigidos a situar a los gobiernos, tanto nacionales como europeos, en una posición que les hace más responsables ante la disciplina del mercado que ante las fuerzas y procesos democráticos. Gill y Cutler subrayan que el nuevo constitucionalismo es parte de un proyecto supremacista, no hegemónico (Gill y Cutler, 2014:14). Los autores definen la supremacía como el gobierno de un grupo de fuerzas que defienden claramente unos intereses concretos al que los grupos subordinados experimentan como un gobierno coercitivo y corrupto que carece de legitimidad y credibilidad (Gill y Cutler, 2014:14). Por tanto, estaríamos ante un bloque no hegemónico, cuyo desarrollo es paralelo a la aparición de un sistema transnacional de libre mercado que depende de un conjunto de complejos estado-sociedad para su funcionamiento, que ejercería la dominación durante un período, sobre una población aparentemente fragmentada, hasta que apareciera una forma coherente de oposición (Gill 2008: 125-126).

\section{GAS Y ACERO CALIENTAN LA GUERRA FRÍA}

Stephen Gill ha destacado el modo en que la reconstrucción económica estuvo vinculada a una reconstrucción política que buscaba reestructurar y asegurar (a través de la OTAN) el capitalismo europeo (Gill, 2008: 59). Los esfuerzos dirigidos a conseguir la hegemonía en las relaciones transatlánticas de posguerra, permitieron, como sostiene el autor, la instauración del capitalismo liberal-socialdemócrata en Europa Occidental evitando que se produjeran revoluciones de izquierdas (Gill, 2008: 59). Este período, que se prolongó desde la década de 1950 hasta el

4 El concepto de hegemonía, desde una perspectiva neo-gramsciana, apunta a un modo de gobernanza basado en un conjunto de normas y prácticas institucionalizadas que, a pesar de ser aceptadas libremente por los grupos subordinados, reflejan una estructura de dominación debido a la asimetría de poder que caracteriza las relaciones entre clases. 
Moreno, Ana. Capital transnacional y gobernanza energética en la Unión Europea. El caso del gas natural.

comienzo de la de 1970, se caracterizó por el modelo de producción fordista, las políticas neo-mercantilistas proindustriales, el desarrollo del estado del bienestar keynesiano y por la reliberalización del plano internacional en su vertiente industrial (Overbeek, 2004: 8; Gill, 2008: 60). El orden social se sustentaba en el compromiso de clase entre las personas trabajadoras y el capitalismo nacional industrial. El compromiso de clase y el corporativismo fueron fundamentales en la construcción del bloque histórico hegemónico durante este período.

El concepto de bloque histórico, en sentido gramsciano, se refiere a la organización de una alianza de diferentes fuerzas de clase en torno a un conjunto de ideas hegemónicas que le proporcionan una dirección estratégica (Gill, 2008: 60). La hegemonía fue producto de nuevos acuerdos sociales relativamente inclusivos. La fracción de clase que lideraba el proceso, el capital productivo, gestionó su hegemonía consensuando el estado del bienestar con las clases subordinadas. La legitimación material del modelo se produjo a través del crecimiento del consumo de masas (Gill, 2008: 60). Se articulaba así el concepto de Occidente en contraposición al grupo de países comunistas. Sin embargo, esta estructura bipolar no impidió el desarrollo de las relaciones alrededor del gas en tanto que los intereses de la fracción de clase que lideraba el proceso en Europa, el capitalismo industrial, precisaba de su evolución.

Los primeros grandes gasoductos transnacionales de Europa occidental encajaban dentro de la lógica política del momento: transportaban gas natural desde el campo de Groningen en Holanda, hacia Bélgica y Alemania desde 1966 y hacia Francia desde 1967. Pero pronto la lógica política en la elección de socios dio paso al dominio de los factores económicos y geográficos. El gas del Sahara se presentaba como una opción de importación para Europa (Högselius et al., 2010: 5). Pero junto a Holanda y el Sahara, comenzaron a considerarse las importaciones de gas soviético a través del telón de acero. Las relaciones para el comercio de gas entre la URSS y Europa occidental comenzaron durante la Guerra fría. De hecho: "several West European countries and regions were connected with the communist pipeline system of Eastern Europe before linking up with the grids of other EC and NATO member states" (Högselius, 2013: 3). La gradual profundización en el comercio de gas y en la construcción de gasoductos parecen enfrentar la lógica fundamental de la Guerra fría. Lo importante aquí es la forma en que un conjunto de intereses muy poderosos y organizados consiguen vencer las resistencias que planteaba un orden construido sobre las bases de la oposición y confrontación política entre el mundo capitalista y el comunista.

En la década de 1960 se descubrieron grandes cantidades de gas natural en la Unión Soviética, pero el crecimiento del sistema de gas soviético estaba condicionado a la capacidad de su industria para producir tubo de acero de alta calidad. La tecnología de la poderosa industria del acero en Europa occidental permitía la fabricación de estos tubos. Sin embargo, la región carecía de grandes reservas de gas que impulsaran su manufactura. Las primeras interesadas en importar gas soviético fueron las petrogasistas estatales ÖMV y ENI, de Austria e Italia respectivamente. Ambas buscaron integrar en los acuerdos los intereses de de una de las industrias que más importancia ha tenido en Europa: el acero. 
Moreno, Ana. Capital transnacional y gobernanza energética en la Unión Europea. El caso del gas natural.

Austria y la URSS alcanzaron un principio de acuerdo en 1966 con un pacto que involucraba a VÖEST, la empresa austriaca del acero, en colaboración con las alemanas Thyssen y Mannesmann, bloqueadas por el embargo de la OTAN sobre las exportaciones de acero a la URSS ${ }^{5}$. VÖEST actuaría como intermediaria para las tuberías alemanas a cambio de que la hoja de metal de VOEST se utilizara como principal producto intermedio en la manufactura de los tubos (Högselius, 2013: 65).

A partir de ese mismo año, con el giro de Alemania hacia la socialdemocracia y el levantamiento formal de la OTAN del embargo sobre las exportaciones de tubo de acero de gran diámetro al bloque soviético, las grandes del acero alemán comenzaron a presionar para conseguir un acuerdo sobre el gas en la línea del austriaco. (Högselius, 2013: 79)

Aunque Bayerngas, cuyo principal accionista era la ciudad de Munich, fue la gasista que comenzó las conversaciones con la URSS, el gobierno federal propuso a Ruhrgas, controlada por Shell y Esso y por los intereses de la industria del carbón alemana, para liderar el proceso (Högselius, 2013: 111). De esta forma se avanzaban los intereses del capitalismo industrial nacional, representado por el carbón y el acero alemanes y al mismo tiempo se favorecía a las transnacionales que, gracias al Estado, se habían situado dentro del acuerdo, consiguiendo un cierto control sobre la competencia tanto dentro del sector del gas, como del gas frente al petróleo.

A medida que avanzaban las conversaciones germano-soviéticas las negociaciones con Francia e Italia se renovaron. El gasoducto (transeuropeo) necesitaba la participación de los tres países para no resultar antieconómico. Además, se reforzarían entre sí como importadores frente a un exportador de la talla de la URSS. (Högselius, 2013: 126). El Gobierno francés, a este respecto, planteó la opción de involucrar a Bruselas que por aquel entonces se encontraba preparando la "common energy policy". No obstante, la idea se rechazó por las dificultades que podrían surgir para alcanzar una posición común. (Högselius, 2013: 127) Alemania occidental comenzó a recibir gas soviético al final de 1973, Italia y Finlandia en 1974 y Francia en 1976 a través de Alemania.

Así pues, el modelo gasístico de la UE echaba a andar en un escenario en el que la protección y el impulso de las poderosas industrias nacionales, que en esos momentos comenzaban a sufrir las consecuencias de la competencia exterior, terminó imponiéndose a la estructura de enfrentamiento con el bloque soviético. El modelo se configuró sobre un elemento clave: los contratos bilaterales de largo plazo (unos 20 años), con precios indexados a ciertos productos petrolíferos y clausula take or pay. Su origen hay que buscarlo en los contratos firmados entre la holandesa NAM y las compañías importadoras de Alemania, Bélgica y Francia. Dado que Shell y Esso eran las accionistas mayoritarias tanto de NAM como de algunas de las importadoras, tuvieron una gran influencia en su construcción. Más tarde, los contratos de exportación del gas holandés se convirtieron en el modelo para las relaciones transnacionales del gas en Europa firmados principalmente con la URSS

5 En general, durante este período, el embargo se consideraba obsoleto no obstante, Alemania representaba una excepción. 
Moreno, Ana. Capital transnacional y gobernanza energética en la Unión Europea. El caso del gas natural.

(y después Rusia) Argelia y Noruega que prácticamente sumaban la totalidad de las importaciones desde fuera del territorio a través de sus compañías estatales Gazprom, Sonatrach y Statoil (Fernández y Palazuelos, 2014: 3).

De igual forma hay que destacar que el modelo se forjó durante un momento de retroceso en la integración europea. Para entender los acontecimientos que siguieron, entre ellos la liberalización, hay que encuadrarlos en el contexto del relanzamiento de la integración y consecuentemente en la batalla política, entre las fuerzas sociales, por la definición del contenido social de esta integración en todos los niveles de la política europea (Van Apeldoorn, 2000: 166). La Directiva sobre el Mercado Interior del Gas (98/30/CE) forma parte de este proceso, y sólo el análisis de la situación puede ayudarnos a esclarecer el hecho de que la Directiva se aprobara pese al rechazo que suscitaba en casi todos los actores con derecho a veto.

\section{EL MERCADO ÚNICO COMO MOTOR DE LA LIBERALIZACIÓN DEL SECTOR ENERGÉTICO}

Como ha sido identificado (Van Apedoorn, 2000: 166-7) el relanzamiento de la integración europea en la década de 1980, relacionado con el planteamiento del mercado único, contenía tres proyectos rivales: un proyecto neoliberal, que concebía el proceso de integración como una oportunidad para abrir Europa a la economía globalizada donde los beneficios del mercado interno se relacionan con la desregulación y las privatizaciones dentro del territorio; un proyecto neomercantilista, para el que el mercado interno constituye un espacio en el que aquellos que lograran constituirse como los "campeones europeos" podrían afrontar con éxito la creciente competencia exterior gracias a mayores economías de escala, y finalmente un proyecto socialdemócrata, que buscaba proteger y consolidar el modelo social europeo. Los dos primeros se identifican con las dos facciones rivales dentro de una emergente clase capitalista transnacional en Europa.

Desde el principio de la década de 1980, distintas organizaciones comenzaron a plantear sus diseños para la construcción del mercado único. La poderosa UNICE (ahora BusinessEurope), pero también la Cámara de Comercio Americana en Bruselas o la ERT presentaban sus propuestas. Esta última se suele referir como la que ejerció mayor influencia en el proyecto final (Van Apeldoorn, 2000; ver también por ejemplo Egan, 2001: 112) y de la que se ha dicho que inspiró el Libro Blanco de la Comisión de junio de 1985, "La consecución del mercado interior" (Van Apeldoorn, 2000: 168). Hay que señalar, sin embargo, que en aquellos momentos las organizaciones europeas estaban más influidas por la fracción neo-mercantilista del capital, y aún así, el Libro Blanco planteaba un mínimo de medidas proteccionistas y estaba más dirigido a la integración europea en la economía global (Van Apeldoorn, 2000: 168). Así, el documento refleja la influencia que las fuerzas sociales que defendían el proyecto neoliberal iban ganando en la construcción europea.

Hasta el momento, no se había establecido en Europa una política común de energía principalmente debido al enfoque que se le aplicaba a esta cuestión desde los estados miembros. La agenda estaba dirigida principalmente hacia la seguridad 
Moreno, Ana. Capital transnacional y gobernanza energética en la Unión Europea. El caso del gas natural.

del suministro debido a una percepción de inseguridad energética. El agotamiento de los recursos se contemplaba como una posibilidad derivada del incremento del consumo. Además, los acontecimientos que habían marcado el suministro externo (el cierre de Suez en 1956 y 1967 y las dos crisis del petróleo de la década de 1970) aumentaban la sensación de inseguridad energética (Herweg, 2015: 92). Pero lo más importante es que esta concepción había dado lugar, en casi todos los estados miembros, a la gestión de la energía a través de monopolios estatales blindados a través de contratos de largo plazo.

Sin embargo, el Libro Blanco y su consecuencia, el Acta Única Europea (1986) ofrecían a la Comisión la oportunidad de impulsar la política energética común. En el Acta Única Europea se establecía la categoría de la libre circulación debido a la anulación de las barreras entre los estados miembros. Aunque no se hacía referencia a la energía, sí permitía plantear un giro en el enfoque de la cuestión. Así, lo que hasta ese momento había sido un problema de seguridad, pasaba a presentarse como un problema de competencia (Herweg, 2015: 93). A este respecto, se criticaban tanto las características monopólicas de los mercados nacionales, como las desventajas que estos monopolios suponían para la competitividad de los grandes consumidores de energía (Herweg, 2015: 93). En este sentido la Comisión disponía del recurso al artículo 90 del Tratado Constitutivo de la CEE, que le permitía formular una Directiva de manera unilateral (Herweg, 2015: 95). Una amenaza que debía ser tenida en cuenta porque para entonces el Tribunal de Justicia Europeo ya había sancionado el uso del artículo 90 por parte de la Comisión en el contexto de la liberalización de las telecomunicaciones (Herweg, 2015: 96).

Conviene en este punto hacer una referencia a la Comisión y sus miembros durante este período. En 1985, cuando Delors llegó a la presidencia de la Comisión, Peter Sutherland $^{6}$, un neoliberal firme defensor del libre mercado (Buch-Hansen y Wigger, 2011: 80) se ponía al frente de la Comisión de Competencia. Como ya se ha indicado, el discurso neoliberal comenzaba a destacar en la economía política europea, sin embargo, las posiciones neo-mercantilistas aún no se habían abandonado por completo. Sutherland estaba determinado a forzar el mercado interior de la energía usando el artículo 90 si llegara a ser necesario (Herweg, 2015: 94). Su sucesor en 1989, en la segunda Comisión Delors, Sir Leon Brittan ${ }^{7}$, seguía el camino marcado por Sutherland y llegó a abrir procedimientos de infracción contra los monopolios de importación y exportación del gas y la electricidad. En cuanto a la

\footnotetext{
6 Sutherland, después de dejar la Comisión, fue Director General del GATT (y después, primer Director General de la Organización Mundial del Comercio) en 1993 y como tal, presidió la Ronda de Uruguay. En el mundo empresarial, Sutherland salió de la Comisión para incorporarse como Presidente al Allied Irish Banks entre 1989 y 1993; fue presidente no ejecutivo de Goldman Sachs International hasta 2015, presidente no ejecutivo de BP hasta 2009, Director del Royal Bank of Scotland Group y miembro del consejo de ABB. En cuanto a las redes de generación de ideas y formación de consenso a las que me he referido en el marco teórico, Sutherland tiene un largo historial, destacaré su pertenencia al Comité Directivo del Grupo Bilderberg hasta 2014, su presidencia de la Comisión Trilateral entre 2001 y 2010 (actualmente es Presidente Honorario) y su vicepresidencia en la European Round Table of Industrialists entre 2006 y 2009.

7 Sir Leon Brittan sirvió en los Gobiernos de Margaret Thatcher desde 1979 a 1986. Después de formar parte de la Comisión Europea, fue vicepresidente de UBS AG Investment Bank, Director no ejecutivo de Unilever y miembro del comité consultivo internacional de Total.
} 
Moreno, Ana. Capital transnacional y gobernanza energética en la Unión Europea. El caso del gas natural.

Comisión de Energía, durante el primer mandato de Delors, estuvo ocupada por Nicolas Mosar, que seguía una línea de negociación política mientras que su sucesor Cardoso e Cunha daba preferencia al acuerdo político, aunque respaldaba también la postura de Sir Leon Brittan (Herweg, 2015: 94). En la tercera Comisión Delors, Abel Matutes sustituyó a Cardoso e Cunha en la Comisión de Energía. Después Marcelino Oreja ${ }^{8}$ y más tarde, Christos Papoutsis ocuparon el puesto, los tres mostraron intenciones negociadoras.

Sin embargo, la Comisión de competencia fue ocupada por Karel van Miert ${ }^{9}$ que, pese a su afiliación socialista, seguía los pasos de Brittan. Continuó con los procedimientos de infracción que éste había iniciado. Durante este proceso, el Tribunal de Justicia aprobo una resolución según la cual la electricidad debía ser tratada como un bien (Herweg, 2015: 100). Esto suponía que la ley permitía la aplicación de las normas de la competencia sobre la electricidad. Así pues, reducida la capacidad de los estados para oponerse, la Comisión se centró en la Directiva sobre la electricidad (Herweg, 2015: 100). Para llegar a un acuerdo sobre el gas fue necesaria esta la experiencia previa.

\section{LIBERALIZACIÓN DEL GAS NATURAL: PRINCIPALES OBJETIVOS Y CAMBIOS}

La Directiva se plantea, por tanto, en un contexto en que la línea neoliberal ha adquirido primacía en la arena europea. El neoliberalismo en Europa fue evolucionando a partir de la década de 1980 como un contraproyecto al orden socioeconómico del liberalismo corporativo en el que el capital industrial gozaba de cierta independencia respecto al capital financiero. (Wigger y Buch-Hansen, 2012: 28) Sus principales ideas se venían desarrollando en ciertos círculos desde las anteriores décadas, pero fue la crisis del sistema lo que le permitió adquirir prominencia. La incorporación de estas ideas a los gobiernos de Reagan y Margaret Thatcher permitió su expansión hacia el continente en el nivel estatal, pero fundamentalmente en la Comisión Europea (Wigger y Buch-Hansen, 2012: 30). Su posterior desarrollo se entiende dentro del marco de lo que Cox (1992 ${ }^{\mathrm{a}}$ : 31) ha planteado como un proceso de internacionalización del estado según el cual, estos se convierten en agencias para el ajuste de las prácticas y las políticas económicas a las exigencias percibidas de la economía global. Estas últimas emanan del consenso de una clase dirigente transnacional situada en las altas esferas del FMI, el Banco Mundial o la OCDE entre otras organizaciones (Overbeek, 2004: 11).

Las nuevas tecnologías del transporte, la comunicación y la información facilitaban el avance del proyecto neoliberal al permitir un rápido proceso de internacionalización del capital tanto productivo como financiero. El poder que el capital transnacional extraía de su movilidad le permitió promover el neoliberalismo en su vertiente política (Wigger y Buch-Hansen, 2012: 29). La internacionalización fue impulsada entonces por sucesivas liberalizaciones y desregulaciones dirigidas a subordinar las

\footnotetext{
8 Cuyo hijo, Marcelino Oreja Arburúa, es actualmente Consejero Delegado de Enagas, la empresa que controla la red de distribución española.

9 Al dejar la Comisión formó parte de los consejos de Anglo-American plc, Philips Lighting BV, Vivendi Universal, RWE, Agfa-Gevaert NV, Goldman Sachs, entre otros.
} 
Moreno, Ana. Capital transnacional y gobernanza energética en la Unión Europea. El caso del gas natural.

economías nacionales, así como la economía global a la disciplina del mercado. (Overbeek, 2004: 9). Todo ello se tradujo en un amplio proceso de desindustrialización, reducción de los costes laborales y una creciente transnacionalización de las estructuras de propiedad y de los circuitos de producción como consecuencia de las subcontrataciones y los procesos de externalización. De este modo comenzaba la transición hacia el postfordismo (Wigger y Buch-Hansen, 2012: 29).

Es en este contexto en el que la Directiva de liberalización del gas natural termina por concretar las tesis sobre la idoneidad de los mecanismos del mercado y de los agentes privados, para garantizar el suministro de energía, que se había gestado en los círculos neoliberales. Así, la argumentación en este sentido se refería a una oferta energética cuya garantía pasaba de ser responsabilidad de los gobiernos a ser una responsabilidad compartida entre aquellos que actuaban en los mercados (Palazuelos y Vara, 2008a: 96). Por otra parte, el discurso subrayaba el modo en que el estímulo de la competencia entre un número creciente de empresas fomentaría una serie de beneficios para los consumidores, principalmente la reducción de los precios de la energía, que llevarían a la mejora de la competitividad de las empresas europeas en los mercados internacionales (Palazuelos y Vara, 2008a: 116).

Las líneas que se marcaron para conseguir estos objetivos en las sucesivas Directivas fueron: el derecho de acceso a las infraestructuras de transmisión de nuevos operadores en 1998; la libertad de los consumidores para elegir sus proveedores y la separación de las actividades de la red de transporte del resto de las actividades relacionadas con la industria del gas en 2003, y finalmente la separación de la propiedad de las empresas que realizaran las actividades de producción, transmisión y distribución en 2009. El proceso de implementación de estas medidas ha provocado importantes cambios en el sector. Como señalan Fernández y Palazuelos (2014: 14), la modificación más importante que ha tenido lugar en el mercado ha sido la gradual introducción y desarrollo de los hubs, que representan el eje del proyecto de la UE para construir una red europea de mercados spot. Estos, introducen los futuros en el mercado del gas y así su financiarización.

La financiarización puede suponer problemas para la seguridad del suministro dado que "desvía las preocupaciones de los operadores del sector y atrae el interés de intermediarios guiados por exclusivos fines especulativos, lo cual origina el debilitamiento de las inversiones y provoca un menor crecimiento de la producción y el deterioro de las infraestructuras" (Palazuelos y Vara, 2008b: 428). Todo ello implica una integración del mercado del gas que facilita la extracción de beneficios a ciertos operadores privados, de manera que la seguridad del suministro energético podría quedar vinculada a la lógica del mercado frecuentemente especulativa.

La introducción de los hubs y su proliferación está relacionada con diferentes cuestiones: el avance de la tecnología en el campo del gas natural licuado (GNL) y las mayores cantidades disponibles en el mercado spot; con el crecimiento del mercado hub en el Reino Unido y de la influencia que éste ejerce en el mercado 
Moreno, Ana. Capital transnacional y gobernanza energética en la Unión Europea. El caso del gas natural.

continental, y finalmente, con el aumento de los precios que experimentó el gas indexado al petróleo a partir de los primeros años de la década del 2000, unido a una reducción del consumo vinculada a la crisis económica a partir de 2008 (Fernández y Palazuelos, 2014: 7-8). Como consecuencia de su implantación, los contratos de largo plazo, firmados con las exportadoras tradicionales, han flexibilizado su estructura: se han eliminado las cláusulas que impedían a los importadores reexportar gas a terceros países, y se han disminuido el tiempo y las condiciones para el ajuste de los precios. Finalmente, el mercado hub ha promovido el desarrollo de un sistema de precios basado en la competencia gas-gas en vez de la indexación al petróleo vinculada a los contratos de largo plazo (Fernández y Palazuelos, 2014: 8-9).

Precisamente, la reducción y la convergencia de precios en el ámbito de la UE era otro de los objetivos declarados de la reforma. Lo cierto es que el objetivo, en lo que se refiere a las familias, no se ha conseguido.

\section{Cuadro 1:}

\begin{tabular}{l} 
Gas prices by type of user \\
EUR per gigajoule \\
Medium size households \\
\hline geo \\
\hline EU ( 28 countries) \\
EU ( 27 countries)
\end{tabular}

\begin{tabular}{|c|c|c|c|c|c|c|c|c|c|c|c|}
\hline : & : & : & : & 14.86 & 16.17 & 14.41 & 15.58 & 17.36 & 18.18 & 18.5 & 18.41 \\
\hline 12.55 & 13.36 & 15.33 & 16.98 & 17.02 & 18.48 & 15.9 & 17.22 & 19.1 & 20.21 & 20.18 & 19.67 \\
\hline
\end{tabular}

Nada impide, sin embargo, que la reducción de precios se produzca en un futuro ya sea por las reformas de la UE o por la evolución en el escenario global. No obstante, la mirada a los precios no deja de ser una mirada unidimensional. Esto es, porque como se ha expuesto, la liberalización contiene muy diversas vertientes: la bajada de los precios de un producto como consecuencia de la competencia podría convertirse en una de ellas. Pero aún así, habría que integrar en los resultados cuestiones como el desempleo o la concentración económica que conlleva. Así pues, de acuerdo con Wigger y Buch-Hansen, los verdaderos beneficiarios de la competencia neoliberal son las compañías que son capaces de imponer los estándares de competición al resto (Wigger y Buch-Hansen, 2012: 35). Respecto a las familias, cuyo poder y capacidad de organización a un nivel transnacional es obviamente mucho menor que el del gran capital, lo cierto es que 54 millones de personas en la UE sufrían pobreza energética en 2015, según las estimaciones del informe recientemente publicado por la Comisión (Pye et al., 2015: v). 
Moreno, Ana. Capital transnacional y gobernanza energética en la Unión Europea. El caso del gas natural.

La liberalización del mercado del gas, por otra parte, dio un impulso a una tendencia ya iniciada con la entrada en vigor del mercado único: la privatización de las empresas de la energía, un sector que había sido tradicionalmente estatal en gran parte de Europa. La defensa de este sistema, basado en el interés de los inversores y la maximización del valor de las acciones, suele basarse en el aumento de la eficiencia. Otra de sus consecuencias, sin embargo, es el modo en que, como sostiene Gill, la privatización, la liberalización y la desregulación son instrumentos que refuerzan un conjunto concreto de intereses de clase, principalmente el poder de los inversores privados (Gill, 2008: 153). La liberalización y la privatización van asociadas al proceso, anteriormente referido, del nuevo constitucionalismo. En este marco, su función consiste en convertir al sector privado y a las grandes corporaciones en los actores principales de la gobernanza global (Gill, 2008: 162).

Tabla 1: Privatizaciones, totales o parciales, llevadas a cabo durante los primeros años del Mercado Único.

\begin{tabular}{|l|l|l|l|}
\hline País & Empresa & Período & Porcentaje \\
\hline Reino Unido & British Gas & 1986 & 100 \\
\hline Austria & OMV & $1987-1996$ & $15+20+14,9+?$ \\
\hline Portugal & Petrogal & $1992-1995$ & $25+?$ \\
\hline España & Enagas & 1994 & 91 \\
\hline Bélgica & Distrigaz & $1994-1996$ & $67+16,62$ \\
\hline Italia & ENI & $1995-1998$ & $15+14+18+15,4$ \\
\hline España & Gas Natural* & 1996 & 3,8 \\
\hline Alemania & $\begin{array}{l}\text { Berliner Gaswerke } \\
\text { (Gasag) }\end{array}$ & 1998 & 51,2 \\
\hline Finlandia & Fortum & $1998-2002$ & $22+9$ \\
\hline
\end{tabular}

Fuente: Elaboración propia a partir de Megginson, 2005: 352-6

Las privatizaciones comenzaron en la década de 1980 con la entrada en vigor del mercado único y han continuado hasta el día de hoy. Las grandes importadoras, anteriormente propiedad de los estados (excepto Ruhrgas), han pasado en gran parte a manos de inversores privados. Así, Italia conserva un 30\% de ENI y Francia un $32,76 \%$ de Engie $^{10}$ (antes Gaz de France y GDF Suez). En un segundo nivel de importancia, Distrigas fue adquirida por ENI y Austria conserva una participación de $1 / 3$ en OMV, el $24,9 \%$ es propiedad de International Petroleum Investment Company (Abu Dhabi). La polaca PKN está participada en menos de $1 / 3$ por el Estado. Portugal posee sólo el $7 \%$ de GALP, mientras que el $38 \%$ es propiedad de Amorim energía, participada por Americo Amorin, Isabel Dos Santos y Sonagol. Finalmente, la danesa Dong vendió recientemente el $18 \%$ a Goldman Sachs y prepara en la actualidad una oferta pública inicial de la que Goldman Sachs ha quedado excluida;

10 Engie, Shareholder Structure http://www.engie.com/en/shareholders/engie-share/shareholder-structure/ (consultado el 02-04-2016). 
Moreno, Ana. Capital transnacional y gobernanza energética en la Unión Europea. El caso del gas natural.

la griega DEPA se encuentra en vías de privatización ${ }^{11}$, y la eslovena Geoplin está incluida en los planes de privatización del Gobierno para $2016^{12}$

Si entre las intenciones de la reforma de la Comisión se encontraba romper con la estructura oligopólica del modelo europeo, lo cierto es que estos "not only persist but deepen" (Fernández y Palazuelos, 2014: 14). Como consecuencia de las privatizaciones, se han producido sucesivas fusiones y adquisiciones que han tenido como resultado la convergencia entre los sectores del gas y la electricidad en grandes grupos de energía. La misma dinámica ha llevado a las grandes importadoras europeas a buscar la integración horizontal, a través de la compra de otros operadores y de la creación de subsidiarias que se ocupan del comercio y la distribución en terceros países, y también la integración vertical, participando en la exploración, producción y proyectos de transporte (Fernández y Palazuelos, 2014: 12).

Por otra parte, como han subrayado Palazuelos y Vara, en la nueva estructura empresarial del sector energético,

"el statu quo basado en el dominio que en cada mercado nacional ejercían monopolios estatales o bien oligopolios sometidos a un fuerte control estatal está siendo sustituido por una estructura empresarial a escala europea sustentada en oligopolios -privados o públicoscuyas actividades son escasamente reguladas por los poderes públicos nacionales y europeos" (Palazuelos y Vara, 2008b: 430).

De esta manera se ha impuesto una mayor limitación a los procesos de participación democrática en un aspecto tan fundamental para la economía como la energía.

\section{CONCLUSIONES}

El desarrollo de la liberalización del sector del gas en la UE no puede explicarse desde la noción neorrealista del estado como actor central en la arena internacional ni desde el planteamiento liberal de la subordinación de la actividad de los actores transnacionales a las iniciativas de las instituciones. Este estudio proponía recorrer el proceso a través del cual se ha construido la estrategia política europea con respecto al sector del gas con el fin de descubrir su origen social. Nos interesaba conocer el papel que han jugado los distintos grupos sociales. En este sentido, se planteaba una arena política formada por la UE y los EEMM en la que las distintas fuerzas sociales promueven sus estrategias. Pero a la vez, se reconocía esta arena como un lugar estructuralmente predispuesto a seleccionar las estrategias de unas clases (o fracciones de clase) sobre las de otras.

Así, el período de postguerra, estuvo caracterizado por un liderazgo, en la economía política europea, del capital nacional productivo que consiguió el respaldo de la clase trabajadora. Pero también por una estructura bipolar del sistema internacional. Sin embargo, ésta no impidió el progreso de las relaciones alrededor del gas en tanto que los intereses de la fracción de clase que lideraba el proceso en Europa, el

11 En vías de privatización: "PPC, DEPA to be placed in new privatization fund subsidiary" en energypress, 1603-2016, disponible en http://energypress.eu/ppc-depa-to-be-included-in-new-privatization-fund-subsidiary/ (consultado el 02-04-2016)

12 "Company profiles - gas trader Geoplin", en STA, 05-04-2016, disponible en https://english.sta.si/2249177/company-profiles-gas-trader-geoplin (consultado el 23-04-2016) 
Moreno, Ana. Capital transnacional y gobernanza energética en la Unión Europea. El caso del gas natural.

capitalismo industrial, precisaba de su evolución. Así, mientras que en un principio los gasoductos transnacionales de Europa occidental encajaban dentro de la lógica de la Guerra Fría, un conjunto de intereses muy poderosos y organizados consiguieron vencer las resistencias que se planteaban para la superación de la división Este-Oeste.

El modelo gasístico se configuró sobre un elemento clave: los contratos bilaterales de largo plazo, con precios indexados a ciertos productos petrolíferos y clausula take or pay. Construido durante un momento de retroceso en la integración europea, para entender el cambio de modelo y la liberalización, hay que encuadrarlos en el contexto del relanzamiento de la integración y la definición del contenido social de la misma derivada de la batalla política entre las fuerzas sociales. A este respecto, se ha subrayado el papel del Libro Blanco "La consecución del mercado interior" (1985) y del Acta Única Europea (1986), que avanzaban los intereses de la fracción neoliberal de la clase capitalista transnacional frente a la fracción neo-mercantilista.

La Directiva para la liberalización del gas se plantea en un contexto en que la línea neoliberal ya ha adquirido primacía. El discurso subrayaba la reducción de los precios de la energía que cabría esperar del aumento de la competencia. Su puesta en marcha ha provocado cambios importantes en el modelo anterior. Uno de ellos, ha sido el desarrollo de los hubs. Estos han introducido la financiarización del sector, lo cual implica la posibilidad de que la seguridad del suministro energético quede subordinada a una lógica del mercado que puede ser especulativa. Por otra parte, éstos tampoco traen aparejada la reducción de precios, y la realidad es que 54 millones de personas en la UE sufrían pobreza energética en 2015.

Finalmente, la liberalización ha promovido la privatización de un sector que había sido tradicionalmente estatal en gran parte de Europa, acompañada de un gran número de fusiones y adquisiciones que conllevan una importante concentración. Lo importante aquí, es el modo en que la privatización, la liberalización y la desregulación son refuerzan unos intereses de clase concretos, principalmente el poder de los inversores privados y, por otra parte, limitan la participación y el control democrático de la energía. 
Moreno, Ana. Capital transnacional y gobernanza energética en la Unión Europea. El caso del gas natural.

\section{BIBLIOGRAFÍA}

Buch-Hansen, H. y Wigger, A. (2011): The politics of European competition regulation. A critical political economy perspective, Routledge, New York.

Ciuta, F. (2010): "Conceptual notes on energy security: total or banal security?", Security Dialogue, vol. 41, nº 1, 123-144. DOI: 10.1177/0967010610361596.

Correljé, A. y Van der Linde, C. (2006): "Energy supply security and geopolitics: A European perspective", Energy Policy, $\mathrm{n}^{\circ} \mathrm{34}, 532-543$.

Cox, R. (1992a): "Global Perestroika" Socialist Register, vol. 28, 26-43.

Cox, R. (1992b): "Multilateralism and world order", Cox, Robert (1996): Approaches to World Order, Cambridge University Press, Cambridge, 494-523.

De Graaff, N. (2013): Towards a Hybrid Global Energy Order. State-owned oil companies, corporate elite networks and governance, Vrije Universiteit, Amsterdam.

Egan, M. (2001): Constructing a European Market: Standards, Regulation, and Governance, Oxford University Press, Oxford y New York.

Fernandez, R. y Palazuelos, E. (2014): "A Political Economy Approach to the European Union Gas Model: Continuities and Changes", JCMS: Journal of Common Market Studies, vol. 52, $n^{\circ}$ 3, 495-511.

Gill, S. (2008): Power and resistance in the new world order: 2 nd edition, fully revised and updated, Palgrave Macmillan.

Gill, S. y Cutler C. (2014): "New constitutionalism and world order: general introduction", Cutler, Claire y Stephen Gill (eds.), New Constitutionalism and World Order, Cambridge University Press, New York.

Hancock, K. J. y Vivoda, V. (2014): "International political economy: A field born of the OPEC crisis returns to its energy roots", Energy Research \& Social Science $\mathrm{n}^{\circ}$ 1, 206-216.

Herweg, N. (2015): "Against all odds: the liberalisation of the European natural gas market. A multiple streams perspective", Tosun, J. et al. (eds.), Energy policy making in the EU. Building the agenda, Springer, London, 87-106.

Högselius, P. et al. (2010): "Natural gas in Cold War Europe: the making of a critical transnational infrastructure", Draft chapter for the EUROCRIT edited volume.

Högselius, P. (2013): Red Gas Russia and the Origins of European Energy Dependence, Palgrave Macmillan, New York.

Mansson, A. (2014): "Energy, conflict and war: Towards a conceptual framework", Energy Research \& Social Science, $\mathrm{n}^{\circ} 4,106-116$.

Megginson, W. (2005): The financial economics of privatization, Oxford University Press, New York.

Overbeek, H. (2004): Global governance, class, hegemony: A historical materialist perspective, Vrije Universiteit, Amsterdam, $n^{\circ}$ 2004/01.

Overbeek, H. (2010): "Global governance: from Radical transformation to neo-liberal management", International Studies Review, $\mathrm{n}^{\circ}$ 12, 697-702.

Palazuelos, E. y Vara, M. J. (2008a): "Unión Europea: Diferencias entre los sistemas energéticos nacionales y obstáculos para una política energética común", Palazuelos, Enrique (dir.), El petróleo y el gas en la geoestrategia mundial, Akal, Madrid, 95- 126.

Palazuelos, E. y Vara M. J. (2008b): "Las reformas liberalizadoras en el sector energético de la Unión Europea", Revista de Economía Mundial, nº 18, 423- 
Moreno, Ana. Capital transnacional y gobernanza energética en la Unión Europea. El caso del gas natural.

\section{6}

Pye, S. et al. (2015): Energy poverty and vulnerable consumers in the energy sector across the EU: analysis of policies and measures, European Commission, Brussels.

Stoddar, E. (2013): "Reconsidering the ontological foundations of international energy affairs: realist geopolitics, market liberalism and a politico-economic alternative", European Security, vol. 22, $\mathrm{n}^{\circ}$ 4, 437-463. DOI: 10.1080/09662839.2013.775122.

Truscott, P. (2009): "Russia and European energy security", Whitehall Papers, vol. $73, n^{\circ} 1,22-31$.

Van Apeldoorn, B. (2000): "Transnational class agency and European governance: the case of the European Round Table of Industrialists", New Political Economy, vol. 5, $\mathrm{n}^{\circ}$ 2, 157-181.

Van Apeldoorn, B. (2002): Transnational Capitalism and the Struggle over European Integration, Routledge, London y New York.

Van Apeldoorn, B. (2004): "Theorising the transnational: a historical materialist approach", Van Apeldoorn, B. (ed.) Transnational historical materialism: the Amsterdam Political Economy Project. Journal of International Relations and Development, Special Issue, vol. 7, n², 142-176.

Van Apeldoorn, B. y De Graaff, N. (2012): "Corporate Elite Networks and US PostCold War Grand Strategy from Clinton to Obama", European Journal of International Relations, Junio, 1-27.

Van Apeldoorn, B., De Graaff, N. y Overbeek, H. (2012): "The Reconfiguration of the Global State-Capital Nexus", Globalizations, vol. 9, $\mathrm{n}^{\circ} 4$, 471-486, DOI: 10.1080/14747731.2012.699915

Van der Pijl, Kees (1984) The Making Of An Transatlantic Ruling Class, Verso, London.

Wigger, A. y Buch-Hansen, H. (2012): "The unfolding contradictions of neoliberal competition regulation and the global economic crisis: a missed opportunity for change?", Van Apeldoorn, B. y Overbeek, H. (eds.), Neoliberalism in crisis, Palgrave Macmillan, New York, 23-44.

Youngs, R. (2007): "Europe's external energy policy between geopolitics and the market", Disponible en el sitio web de FRIDE http://www.fride.org/publication/295/europe\%27s-external-energy-policy:between-geopolitics-and-the-market. 